\title{
Impact of the level of Temporal and Operational Detail in Energy-System Planning Models
}

\author{
Kris Poncelet ${ }^{\mathrm{a}, \mathrm{b}, \mathrm{c}}$, Erik Delarue ${ }^{\mathrm{a}, \mathrm{c}}$, Daan Six ${ }^{\mathrm{b}, \mathrm{c}}$, Jan Duerinck ${ }^{\mathrm{b}, \mathrm{c}}$, William D'haeseleer ${ }^{\mathrm{a}, \mathrm{c}, *}$ \\ ${ }^{a}$ KU Leuven, Department of Mechanical Engineering, TME Branch, Celestijnenlaan 300, 3001 Heverlee (Leuven), Belgium \\ ${ }^{b}$ VITO, Boeretang 200, $2400 \mathrm{Mol}$, Belgium \\ ${ }^{c}$ Energy Ville, Thor Park, 3600 Genk, Belgium
}

\begin{abstract}
To limit the computational cost, bottom-up long-term (LT) energy-system planning models typically have a stylized temporal representation and do not consider techno-economic operational constraints of power plants. Both these simplifications have been shown to have a significant impact on the results. However, increasing the level of temporal and techno-economic operational detail will result in an increased computational cost. In this regard, the first goal of this work is to quantify which of these simplifications has the highest impact on the results, and should therefore be prioritized for improving. To do so, the impact of both the low level of temporal and techno-economic operational detail are quantified for a varying penetration of intermittent renewable energy sources (IRES). For a high penetration of IRES, the gains obtained by improving the temporal representation are shown to outweigh the gains obtained by incorporating detailed techno-economic operational constraints. Therefore, improving the temporal representation is suggested to be prioritized. The second goal of this paper is to identify opportunities for model improvements. Different approaches to improve the temporal dimension are proposed. While the focus in the literature lies primarily on the impact of the temporal resolution, this work more fundamentally considers and assesses different approaches of dealing with the temporal dimension. Using a different approach of defining the time segments to explicitly account for IRES variability is found to lead to a higher accuracy than can be obtained by simply increasing the temporal resolution, while requiring a lower number of time segments. Moreover, a temporal representation based on selecting a set of representative days can achieve an even higher accuracy, albeit requiring a higher number of time slices. An additional advantage of using representative days is that chronology is retained such that the impact of short-term dynamic fluctuations of IRES can be accounted for.
\end{abstract}

Keywords: Energy system planning, integration of renewable energy sources, TIMES, temporal resolution, operational constraints, power systems

\section{Introduction}

Bottom-up, long-term (LT) energy system planning models are frequently used to analyze pathways for the transition of (parts of) the energy system and to deduce policy advice. In this category of models, popular examples are, amongst others, MARKAL/TIMES, PRIMES, EnergyPLAN, IKARUS and PERSEUS 1]. 5 In recent years, multiple studies have analyzed scenarios for the evolution towards a sustainable energy system, either focusing on the feasibility of realizing ambitious targets for renewable energy or the reduction of greenhouse gas (GHG) emissions (e.g., [2, 3, 4), the role of specific technologies (e.g., [5, ,6]) or the role of policy instruments (e.g., 7]) on the transition pathway.

To decarbonize the energy system, the share of renewable generation is expected to increase significantly, especially in the electricity sector [8]. Some of these renewable energy sources, such as wind energy and solar

*Corresponding author. Tel: +32 163225 10. E-mail addresses: william.dhaeseleer@kuleuven.be 
photovoltaic (PV) energy, have an intermittent character, i.e., they are highly variable and have a limited predictability. Due to these characteristics, a large penetration of intermittent renewable energy sources (IRES) can have a significant impact on the operation of the electric power system. First, the variability of IRES generation increases the need for cycling of dispatchable power plants (i.e., changing the electric 15 power output by ramping up/down or by switching on/off) 9, 10, 11. Second, sufficient back-up capacity is needed to deal with periods in which IRES output is low. Third, the limited predictability of IRES generation leads to an increased demand for operating reserves [12, 13.

Long-term energy system planning models typically use a stylized temporal representation (i.e., a year is typically represented by 1-12 so-called "time slices"). Furthermore, these models typically operate at a 20 technology-type level, rather than considering individual power plants and corresponding technical loadfollowing constraints and cycling costs. This level of detail has so far been reserved for operational models (i.e., unit-commitment (UC) models). Historically, this level of detail could be ignored in planning models without a major impact on the quality of the results [14, 15. However, recently it is becoming more and more clear that the traditional approach used in LT planning models falls short of accurately representing

25 the unit commitment and dispatch for electricity systems with high shares of IRES. In this regard, a myriad of approaches attempting to bridge the gap between planning models and operational models is currently being developed. Two fundamentally different groups of approaches can be identified. A first group of approaches scrutinizes the results of the energy-system planning model with dedicated operational models with the goal to better interpret the results (unidirectional soft-link, e.g., [16]), or to adapt the parameters

30 of the energy system model (biderectional soft-link, e.g., [17, 18]). A second group of approaches aims to directly increase the level of detail in planning models, either focusing on the temporal representation (e.g., [19, 20, 21]) or on the level of techno-economic operational detail (e.g., 14, 22]). Both increasing the level of temporal detail and the level of techno-economic operational detail comes at an increased computational cost.

35 The aim of this work is twofold. The first goal is to determine whether the low level of temporal detail or the low level of techno-economic operational detail has the highest impact on the results, in order to provide guidelines as to which aspect should be improved with the highest priority. To do so, the impact of the low level of temporal and techno-economic operational detail is quantified for a varying penetration of IRES. Second, by analyzing in depth how the level of temporal and techno-economic operational detail 40 impact the results, the aim is to identify and compare different opportunities for improving the operational dimension in planning models.

The remainder of this paper is organized as follows: Section 2 gives an overview of the literature regarding the impact of the level of temporal and techno-economic operational detail used in planning models. Subsequently, Section 3 presents the methodology, the assumptions and the data used for quantifying the 45 impact of the temporal and techno-economic operational representation. The results of this analysis, as well as ways to improve the operational dimension, are discussed in Section 4 Finally, a conclusion and some good practice guidelines are presented in Section 5 .

\section{Literature review}

Different authors have recently investigated the impact of the temporal resolution on the model results. so A first group analyzes the effect of increasing the temporal resolution on balancing electricity demand and supply (i.e., the dispatch, no investment decisions are considered) [16, 20. Using a low temporal resolution is shown to lead to an overestimation of the uptake of IRES. A second group analyzes the impact of the temporal resolution on investment decisions [19, 23, 21. Main results are that by using a low temporal resolution, the optimal level of investments in less flexible baseload technologies and IRES is overestimated, while the optimal level of investments in flexible dispatchable generation technologies is underestimated. Regarding the impact of the level of techno-economic operational detail, Palmintier 14 shows that neglecting operational constraints results in a sub-optimal capacity mix, in turn leading to higher operating costs and carbon emissions. Nweke et al. 24, show in a case study of the South Australian power system that integration of operational constraints in planning models has a significant impact on the investment decisions. Finally, 
Welsch et al. 22 demonstrate in a case study of Ireland that neglecting flexibility requirements strongly impacts the generation portfolio.

While there is some literature on this topic, the existing literature does not allow to answer the question whether the low level of temporal detail or the low level of techno-economic operational detail has the highest impact on the model results for multiple reasons. First, the the existing literature typically focuses either on the impact of the low level of temporal detail or on the impact of the low level of techno-economic operational detail. As these studies are based on very different power systems, the results cannot be easily compared. In addition, in some studies the focus is on the impact on investments, whereas other studies focus on the dispatch. Second, in the majority of studies focusing on the low level of temporal detail (e.g., $[19,21,23]$ ), the impact of this level of detail is quantified by comparing the results of a model with a temporal

to representation typical for long-term planning models with an advanced model that has an increased temporal resolution (i.e., an increased number of diurnal time slices). However, as will be shown in Section 4.3 of this paper, merely increasing the temporal resolution is not sufficient to have an accurate representation of the temporal dimension. Therefore, the used reference of comparison does not allow to quantify the impact of the temporal representation. In contrast, Haydt et al. 20 do use a correct reference for the temporal

75 representation. However, the impact of the low level of temporal detail is quantified for a case study of the Flores island, which is not representative for large electricity systems. Moreover, electricity generation in the Flores island is based on wind turbines, run-of-river hydro and diesel generators. Due to the fact that there is only one source of fully controllable electricity generation, the impact of the low level of temporal detail on the number of operating hours of different types of generators (baseload, mid-merit, peak load) so cannot be analyzed. Finally, a number of studies presenting novel approaches to bridge the gap between planning and operational models address both the temporal and the technical aspect simultaneously (e.g., 16, 22, 24]). However, the focus in these studies is on evaluating the improvements realized by the presented approach, rather than quantifying the impact of the low level of temporal and techno-economic operational detail. A single exception is the work of Deane et al. [16] which allows, to some extent, to analyze the ${ }_{85}$ impact of both aspects separately. However, their analysis is limited to single penetration level of IRES. As will be discussed in Section 4.2 of this paper, the impact of the temporal and techno-economic operational detail is strongly dependent of the penetration level of IRES.

This paper contributes to the existing literature in two ways. First, while the literature typically focuses either on the impact of the temporal aspect, or on the impact of the techno-economic aspect, this work

90 simultaneously addresses both aspects (and the interactions between the two) for a varying penetration of IRES. This allows to make the trade-off between improving planning models by extending the temporal detail and/or aiming for a better technical representation. Second, when developing approaches for reducing the impact of the low level of temporal detail, the literature primarily focuses on increasing the temporal resolution (i.e., the number of diurnal time slices). The work presented in this paper takes a broader 95 perspective and analyses and compares fundamentally different approaches to represent the temporal dimension, thereby providing fundamental insights into how this choice affects main model results, and how the temporal representation can be improved.

\section{Methodology, data and assumptions}

\subsection{Methodology}

In a first step, the aim is to analyze and quantify the impact of the temporal representation and the level of techno-economic operational detail commonly used in large-scale planning models. Quantifying the impact of both these aspects allows to make the trade-off between improving the temporal representation and/or increasing the level of techno-economic operational detail.

The methodology used to quantify the impact of the low level of temporal and techno-economic operational detail is based on the soft-linking methodology as described by Deane et al. [16. The methodology consists of the following steps:

1. Run the LT planning model for a specific scenario with a gradually increasing penetration of IRES. 
2. Extract the results from this model for multiple target years. The results include the generation portfolio, the electricity generation shares of each technology and the operational costs.

3. Convert the installed capacities of each technology in a number of individual power plants, and provide this input data to the operational UC model. For each type of power plant, provide additional technoeconomic characteristics (e.g., minimum stable generation level, minimum up and down times, start-up costs).

4. Provide the original time series for the electricity demand and the availability of IRES for an entire year at an hourly resolution to the operational UC model.

5. Run the UC model without including the detailed techno-economic operational constraints. Without these constraints, the dispatch in every hour will follow the merit-order (MO). Therefore this model is referred to as the $\mathrm{MO}$ dispatch model in the remainder of this paper. Compare the results of this model with those of the LT planning model to analyze the impact of the low level of temporal detail in the LT planning model.

6. Run the UC model with detailed techno-economic operational constraints. Compare the results of this model with those of the MO dispatch model to analyze the impact of the low level of techno-economic operational detail.

7. Repeat steps 5 and 6 for every target year to analyze the relation between the penetration of IRES and the impact of the low level of temporal and techno-economic operational detail.

A schematic overview of the methodology applied is presented in Figure 1.

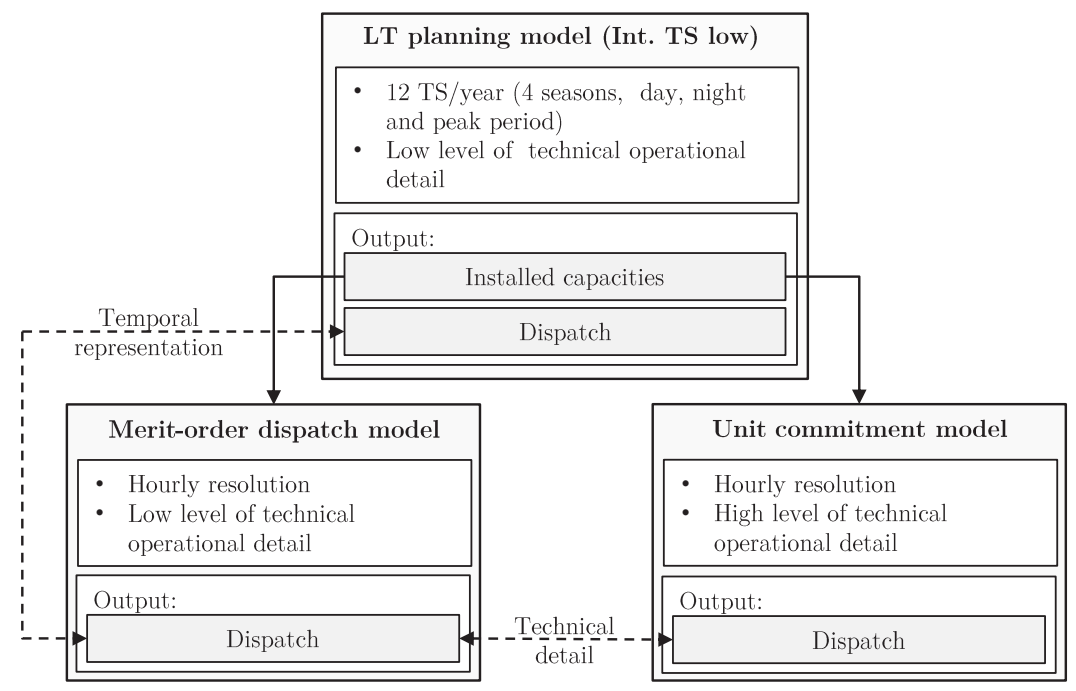

Figure 1: Schematic overview of the methodology.

Once the impact of both the temporal representation and the level of techno-economic operational detail are analyzed and quantified, opportunities for model improvements are identified. Different approaches and their respective strengths and limitations are presented.

\subsection{LT energy-system planning model: TIMES}

The LT model used in this work is generated in the TIMES environment. TIMES (an acronym for The Integrated MARKAL-EFOM System) is an economic model generator for local, national or multiregional energy systems. TIMES models can be classified as bottom-up (technology explicit), dynamic partial equilibrium models of energy markets, typically formulated as LP problems [25]. By simultaneously making investment and operational decisions over a specified time horizon in order to minimize the discounted total system cost, the dynamic evolution of a reference energy system can be analyzed. This reference energy 
system is specified by providing four types of input data: the demand for energy services, the resource potential, a techno-economic description of a set of technologies, and a set of policies. While TIMES models are generally used to analyze scenarios for the evolution of the entire energy system, it can be applied to a single sector as well (e.g., the electricity sector).

Figure 2 gives a schematic overview of how the temporal dimension is modeled in TIMES. The planning horizon is divided into a set of periods, each represented by a single year (so-called "milestone year"). In turn, these milestone years can be divided into a set of time slices, serving to represent intra-annual variations in demand and supply. In the presented example, a year is disaggregated into four seasons, which are in turn disaggregated into weekdays (WD) and weekend (WE) periods. Finally, diurnal variations are introduced via a day $(\mathrm{D})$ and a night $(\mathrm{N})$ time slice, resulting in a total of 16 time slices. The number of time slices within each level of the time-slice tree and the duration of each time slice can be specified freely by the user. However, due to computational restrictions, the number of time slices used in large-scale energy-system planning models is generally restricted to 1-121. Within each time slice, all parameters are fixed, i.e., the load and the availability of IRES are assigned a single value within each time slice. The value assigned to the load or the availability of IRES in every time slice is typically taken as the average value of all data points of the respective time series corresponding to that time slice (e.g., the average demand for electricity in weekend nights during winter is assigned to the time slice corresponding to night periods in the weekends in the winter season) ${ }^{2}$ Following Haydt et al. 20, this approach of assigning values to time slices is referred to as the "Integral method", referring to the fact that all available data is used.

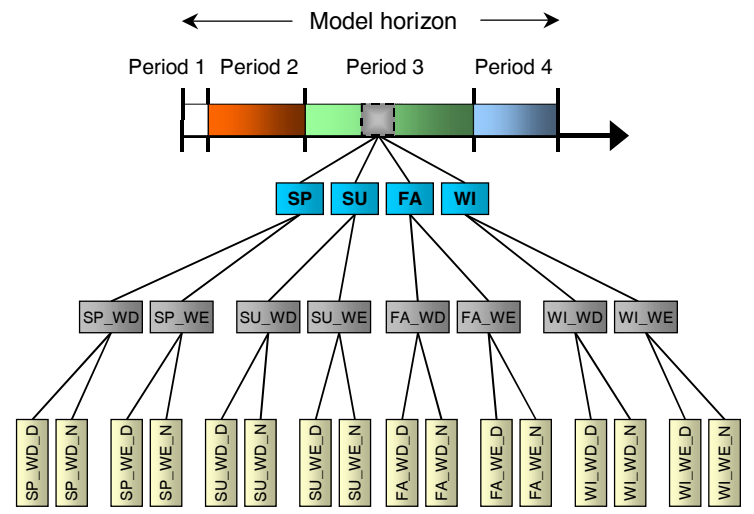

Figure 2: An example of the temporal structure in TIMES models [25].

From a techno-economic perspective, each generation technology is described by an efficiency, an availability factor and an endogenously determined capacity. In terms of operational costs, fuel costs, variable O\&M costs, and taxes are accounted for. Two constraints are essential for the operation of the power system in the model. A first constraint limits the generation $a c t_{p, v, t, s}$ ("activity") in time slice s of year $\mathrm{t}$ of a technology p ("process"), that has been installed in an earlier year v, to the available installed capacity of that technology:

$$
a c t_{p, v, t, s} \leq A F_{p, v, t, s} \cdot A C F R_{p, v, t} \cdot F R_{s} \cdot C A P_{-} A C T_{p} \cdot \operatorname{cap}_{p, v, t} \quad \forall p, v, t, s
$$

The available capacity is limited to a fraction of the total installed capacity $\left(\operatorname{cap}_{p, v, t}\right)$ to account for retirements and lead times (represented by endogenous parameter $A C F R_{p, v, t}$ ). Moreover, to account for periodic

\footnotetext{
${ }^{1}$ In TIMES, the structure of the time-slice tree, and therefore the number of time slices used, can be determined freely by the user. The ranges presented here merely serve to indicate common practice. Recent applications using a higher number of time slices are i.a. 2 . 19 .

${ }^{2}$ It is assumed that time series of load, and IRES availability are available with a high resolution (e.g., hourly or quarterhourly data).
} 
maintenance and forced outages, this available capacity is reduced by the availability factor $A F_{p, v, t, s}$. For conventional power plants, this availability factor is typically taken identical in every time slice. For IRES, the availability factor accounts for both maintenance and the limited availability of the resource (e.g., wind) within each time slice. Finally, $C A P_{-} A C T_{p}$ is a conversion factor from units of capacity (e.g., MW) to units of activity (e.g., MWh), assuming that the capacity is running at full load for the entire year, and $F R_{s}$ is the duration of time slice s expressed as a fraction of the year. The activity of a process is unambiguously linked to a specific commodity flow $\left(f l_{c, p, v, t, s,{ }^{\prime}{ }^{o u t} t^{\prime} /{ }^{\prime} i n^{\prime}}\right)$ entering and/or leaving that process, and linear relationships between the different commodity flows entering or leaving a process are established to specify the efficiency of a process, and the level of emissions related to the activity of a process.

Second, for each commodity, a balance equation, ensuring that consumption does not exceed generation is imposed. For the commodity electricity, the net flow must equal the demand in every time slice:

$$
\sum_{p, v}\left(f l_{c, p, v, t, s,{ }^{\prime} o^{\prime}}-f l o_{c, p, v, t, s,{ }^{\prime}{ }^{\prime} n^{\prime}}\right)(\geq ;=) D E M_{c} \cdot C O M_{-} F R_{c, s} \quad \forall c, t, s
$$

Here $D E M_{c}$ is the yearly demand for commodity c, and $C O M_{-} F R_{c, s}$ is the fraction of this demand in time slice $\mathrm{s}$.

From these constraints, it is clear that the dispatch of power plants in TIMES models follows the meritorder, where the instantaneous generation is only restricted by the available capacity, and not by detailed techno-economic operational constraints.

\subsection{ST operational unit commitment model}

The UC model used in this work is LUSYM. This model determines the optimal scheduling of a given set of power plants to meet the electricity load, taking account of the techno-economic operational constraints of power plants and the electricity system [26. The model applied in this work uses hourly time series for an entire year of data and considers the following techno-economic constraints for individual power plants: minimal stable generation level, minimum up and down times, maximum ramping rate, part-load efficiency losses, maintenance requirements, start-up costs and ramp-costs. A full description of this model can be found in 26 .

\subsection{Data and assumptions}

The TIMES model used in this work is based on the Belgian electricity system. It is assumed here that the Belgium electricity system is representative for other thermally-dominated systems with low potentials for reservoir hydro generation. The time horizon of the planning model is 2014-2055, and is divided into 5 periods. To achieve a varying penetration of IRES throughout the model horizon, a linearly increasing target for the share of annual electric energy generated by IRES is imposed (0\% in 2010, 50\% in 2050). Each milestone year is subdivided in a total of 12 time slices. Four time slices are used to represent seasonal variations. Each seasonal time slice is in turn disaggregated into a day time slice, a night time slice and a time slice corresponding to hours of peak electricity demand 3 . Values are assigned to each time slice following the integral method. In the remainder of this text, this temporal representation is referred to as 'Integral TS low'. The demand for electricity is assumed to be inelastic and inflexible. Besides the electricity demand, a demand for firm capacity, exceeding the annual peak load by $5 \%$, is imposed in the planning model to ensure generation adequacy. Moreover, in all presented models, grids and cross-border trade are disregarded (i.e., island operation with a single node is assumed) and the requirement for operating reserves is disregarded. Finally, a constant discount rate of $5 \%$ is applied.

The set of technologies considered in this work is restricted to conventional dispatchable power plants, IRES and pumped storage plants. The considered dispatchable technologies are third generation nuclear power plants (NUC), subcritical and (ultra-)supercritical pulverized coal plants (SubC PC and (U)SC PC),

\footnotetext{
${ }^{3}$ As the time slices can be defined freely within the TIMES environment, these time-slice divisions differ to some extent from model to model. Similar time-slice divisions use e.g. 4 seasonal and 2 diurnal time slices, or add a time-slice level to separate weekdays from Saturdays and Sundays.
} 
internal gasification combined cycle plants (IGCC), combined cycle gas turbines (CCGT) and regular and advanced open cycle gas turbines (OCGT). The IRES considered are solar PV and onshore and offshore wind turbines. The economic and operational characteristics of these technologies are presented in Tables 1.2. The investment costs, fixed operations and maintenance (FOM) costs and the efficiency are dependent on the timing of the investment, whereas the other characteristics are assumed constant throughout the time horizon. Given the limited geographical potential for additional pumped hydro plants in Belgium, no additional investments in new pumped-storage plants are allowed. Moreover, note that combined heat and power (CHP) plants or small generators that might play a more significant role in future energy systems in the context of increasing distributed generation and the implementation of smart grids are not considered.

\begin{tabular}{|c|c|c|c|c|c|c|c|c|c|c|c|}
\hline \multirow[b]{3}{*}{ Technology } & \multirow{2}{*}{\multicolumn{4}{|c|}{ Investment cost $\left[\mathrm{kEUR} / \mathrm{kW}_{e}\right]$}} & \multirow{2}{*}{\multicolumn{4}{|c|}{$\mathrm{FOM}\left[\mathrm{EUR} /\left(\mathrm{kW}_{e} \cdot\right.\right.$ year $\left.)\right]$}} & \multicolumn{3}{|c|}{ VOM [EUR/MWh] } \\
\hline & & & & & & & & & & Life & Time $[\mathrm{y}]$ \\
\hline & 2010 & 2020 & 2030 & 2050 & 2010 & 2020 & 2030 & 2050 & & & Lead Time $[\mathrm{y}]$ \\
\hline NUC & 3.66 & 3.66 & 3.66 & 3.66 & 0 & 0 & 0 & 0 & 11.1 & 60 & 7 \\
\hline SubC PC & 1.37 & 1.37 & 1.37 & 1.37 & 27 & 27 & 27 & 27 & 7.7 & 35 & 4 \\
\hline (U)SC PC & 1.71 & 1.71 & 1.71 & 1.71 & 34 & 34 & 34 & 34 & 6 & 35 & 4 \\
\hline IGCC & 2.76 & 2.49 & 2.25 & 1.83 & 55 & 50 & 45 & 37 & 7.5 & 30 & 4 \\
\hline CCGT & 0.86 & 0.86 & 0.86 & 0.86 & 26 & 21 & 20 & 20 & 5 & 25 & 2 \\
\hline OCGT & 0.49 & 0.49 & 0.48 & 0.47 & 12 & 12 & 12 & 1 & 4 & 15 & 2 \\
\hline Adv. OCGT & 0.57 & 0.57 & 0.57 & 0.57 & 17 & 17 & 17 & 17 & 4 & 15 & 2 \\
\hline Onsh. $\overline{\text { Wind }} \overline{-}$ & $1.4 \overline{0}$ & $1.2 \overline{7}$ & $\overline{1.1 \overline{9}}$ & $\overline{1} . \overline{1} \overline{1}$ & $3 \overline{4}$ & $2 \overline{7}$ & $2 \overline{4}$ & $\overline{2} \overline{1}$ & - & $\overline{2} \overline{5}$ & 1 \\
\hline Offsh. Wind & 4.30 & 3.40 & 2.70 & 2.10 & 130 & 95 & 75 & 60 & - & 25 & 1 \\
\hline Sol. PV & 3.66 & 1.42 & 1.14 & 0.78 & 51 & 16 & 13 & - & 10 & 30 & 1 \\
\hline
\end{tabular}

Table 1: Economic characteristics of the considered technologies.

\begin{tabular}{|c|c|c|c|c|c|c|c|c|c|}
\hline & & & NUC & SubC PC & (U)SC PC & IGCC & CCGT & OCGT & Adv. OCGT \\
\hline \multirow{4}{*}{ Efficiency } & \multirow{4}{*}[\%]{} & 2010 & 1004 & 37 & 45 & 45 & 58 & 39 & 42 \\
\hline & & 2020 & 100.14 & 38 & 46 & 46 & 60 & 39 & 45 \\
\hline & & 2030 & 1024 & 39 & 49 & 48 & 62 & 40 & 45 \\
\hline & & 2050 & $104 \overline{4}$ & 41 & 49 & 50 & 64 & 41 & 45 \\
\hline$P_{\min }$ & {$\left[\% / P_{\text {nom }}\right]$} & & 50 & 40 & 50 & 50 & 50 & 10 & 10 \\
\hline Eff. loss at $P_{\min }$ & {$[\% \mathrm{pt}]$} & & - & 2 & 2 & 8 & 8 & 21 & 21 \\
\hline Ramp rate & {$\left[\begin{array}{ll}\% & P_{n o m} / \mathrm{min}\end{array}\right]$} & & 5 & 3 & 4 & 4 & 7 & 17.5 & 17.5 \\
\hline Ramp cost & {$[\mathrm{EUR} / \Delta \mathrm{MW}]$} & & - & 1.3 & 1.3 & 0.25 & 0.25 & 0.66 & 0.66 \\
\hline MUT & [hours] & & 24 & 6 & 6 & 4 & 4 & 1 & 1 \\
\hline MDT & [hours] & & 48 & 4 & 4 & 1 & 1 & 1 & 1 \\
\hline Start-up energy & {$\left[M W h_{t h} / \Delta M W_{e}\right]$} & & 17 & 5.7 & 5.7 & 1.7 & 1.7 & 0.02 & 0.02 \\
\hline Start-up depr. & {$[\mathrm{EUR} / \Delta M W]$} & & 1.7 & 5 & 5 & 10 & 10 & 10 & 10 \\
\hline Availability & {$[\%]$} & & 85 & 85 & 85 & 85 & 85 & 85 & 85 \\
\hline
\end{tabular}

Table 2: Operational characteristics of the considered technologies.

With the exception of the nuclear plants, data on investment costs, fixed operations and maintenance (FOM) costs, life times and efficiencies are taken from 27. Data on nuclear plants and lead times are taken from [28. Variable operations and maintenance (VOM) costs and technical characteristics of different technologies are adopted from 29. Regarding IRES, generation profiles for onshore and offshore wind turbines and solar PV panels are taken from measured output in 2013, as provided by the Belgian transmission system operator Elia [30. This generation profile is scaled to the installed capacity in future years. The generation system in the base year (2014), documented by Elia 30, is taken as the current Belgian electricity generation system. The age of existing power plants is assumed to be equally distributed between 0 year and the respective technology's lifetime. Similar to the IRES generation profiles, the profile of future electricity demand is considered to be identical to the one observed in 2013 [30. This profile is scaled using 
a constant electricity demand growth rate of $1 \%$ per year. Fuel prices in the first period are adopted from [28], while fuel price evolutions are derived from [31]. The assumed fuel and emission allowance prices are given in Table 3 .

\begin{tabular}{|c|c|c|c|c|c|}
\hline Price & 2010 & $\begin{array}{c}2020 \\
{[\bar{E} \bar{U}]}\end{array}$ & $\begin{array}{r}2030 \\
-- \\
2008 / \Lambda\end{array}$ & $\begin{array}{r}2040 \\
\left.\bar{W} \bar{h}_{p}\right]\end{array}$ & 2050 \\
\hline Coal & 8.81 & 9.44 & 9.79 & 10.33 & 10.82 \\
\hline Natural gas & 23.89 & 24.30 & 25.12 & 25.66 & 26.27 \\
\hline Uranium[4] & 6.34 & 6.34 & 6.34 & 6.34 & 6.34 \\
\hline & \multicolumn{5}{|c|}{$\left[\bar{E} \bar{U} \bar{R}_{2008}^{-} / \overline{\operatorname{ton}} \bar{C} \bar{O}_{2}^{e \bar{q}}\right]$} \\
\hline GHG emissions & 0 & 12.5 & 25 & 37.5 & 50 \\
\hline
\end{tabular}

Table 3: Fuel and greenhouse gas (GHG) emission prices.

\section{Results and Discussion}

\subsection{Impact of the model simplifications on operational decisions}

Figure 3 presents, for each milestone year, the electric energy generation shares following from the dispatch in the TIMES model, the MO dispatch model, and the UC model. Differences in dispatch between the TIMES model and the MO dispatch model are solely due to the simplified temporal representation in the TIMES model. The difference between the MO dispatch model and the UC model are due to the low level of techno-economic detail in the MO dispatch model. Furthermore, Table 4 displays the generation mix error for the different models 5 . As the UC model has the highest level of detail, it serves as a reference for comparison. At this point, it must be stressed that the aim of this work is not to present scenarios for the evolution of the Belgian electricity system, but rather to analyze the impact of the modeling assumptions typically used in LT planning models. In this regard, our interest lies in the difference in results between the different models, and not in the model results as such.

A first observation is that in the first two periods, there are only slight differences in dispatch. However, as the share of IRES increases, a divergence in the generation shares in the different models starts to appear. This confirms the presumption that the importance of the temporal resolution and the inclusion of operational constraints grows with the level of IRES penetration.

\begin{tabular}{|c|c|c|c|c|c|}
\hline & 2014 & 2020 & 2030 & 2040 & 2050 \\
\hline Generation mix error TIMES [\%] & 0.4 & 0.4 & 6.5 & 10.2 & 12.8 \\
\hline Generation mix error MO dispatch [\%] & 0.4 & 0.4 & 3.5 & 3.1 & 2.4 \\
\hline$\Delta$ generation mix error temporal detail [\%] & 0.0 & 0.0 & 3.0 & 7.1 & $\overline{1} \overline{0} \overline{4}$ \\
\hline$\Delta$ generation mix error techno-economic detail [\%] & 0.4 & 0.4 & 3.5 & 3.1 & 2.4 \\
\hline 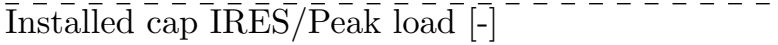 & $\overline{0} \overline{3} \overline{0}$ & $\overline{0} . \overline{4} \overline{2}$ & $\overline{0} \overline{7} \overline{7}$ & $\overline{1} . \overline{1} \overline{2}$ & $\overline{1} . \overline{4} \overline{9}$ \\
\hline Installed cap Nuclear/Peak load [-] & 0.45 & 0.52 & 0.63 & 0.52 & 0.42 \\
\hline
\end{tabular}

Table 4: Generation mix error in the different models.

Two patterns can be observed in the deviations in dispatch (see Figure 3). First, the share of baseload electric energy generation tends to be overestimated by the TIMES model. Second, this model also systematically overestimates the uptake of IRES. In other words, more curtailment of IRES is required/cost-effective

\footnotetext{
${ }^{4}$ Fuel prices for nuclear plants are expressed in $[\mathrm{EUR} / \mathrm{MWh}$ ] and include front-end and back-end costs of the nuclear fuel cycle. Efficiencies for these plants are adjusted to correspond to the fuel prices.

${ }^{5}$ The generation mix error is defined as: GenMixError $=\sum_{i} \frac{\mid \text { generation_share }{ }_{i}-\text { generation_share }_{i}^{U C} \mid}{2}$, where index i runs over all technologies and the generation shares are expressed as a percentage.
} 


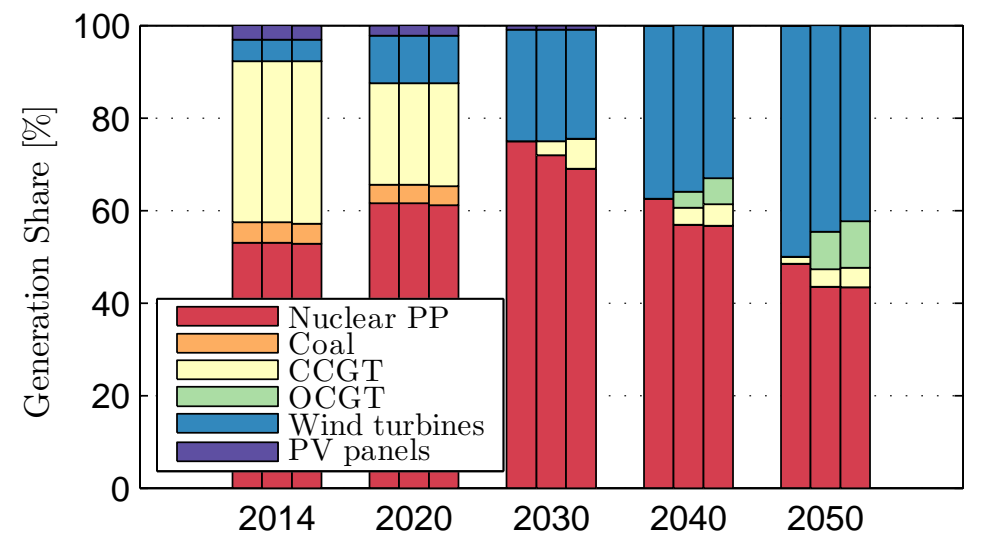

Figure 3: Electric energy generation shares for each milestone year in the different models. The left, middle and right bar respectively correspond to the TIMES model, the merit-order dispatch model and the unit commitment model.

than is anticipated by the TIMES model. A result of this higher level of curtailment is that the proposed portfolio falls short of achieving the imposed target for the share of IRES in the generation mix. An overview of curtailment of IRES and the share of IRES in the generation mix in the different models is presented in Table 5. From Figure 3, it can be observed that both the temporal representation and the low level of techno-economic detail contribute to the overestimation of baseload and IRES generation. This is in line with the findings of [16, 19, 20, 21].

\begin{tabular}{|c|c|c|c|c|c|}
\hline & 2014 & 2020 & 2030 & 2040 & 2050 \\
\hline Curtailment TIMES [\%] & 0 & 0 & 0 & $\overline{0}$ & 0 \\
\hline Curtailment UC $[\%]$ & 0 & 0 & 1.9 & 11.5 & 15.4 \\
\hline 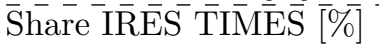 & $\overline{7} . \overline{7}$ & $1 \overline{2} . \overline{5}$ & $\overline{2} 5$ & $3 \overline{7} . \overline{5}$ & 50 \\
\hline Share IRES UC [\%] & 7.7 & 12.5 & 24.5 & 33.1 & 42.3 \\
\hline
\end{tabular}

Table 5: Curtailment of intermittent renewable energy sources (IRES) and shares of IRES generation in the energy mix. Curtailment is expressed as a percentage of the maximal IRES generation (i.e., when there is no curtailment). The share of renewable electricity generation is expressed as a percentage of total consumed electric energy.

The operational costs in the different models are presented in Table 6. The operational costs include fuel costs, costs related to emissions of greenhouse gases, variable operations and maintenance (VOM) costs as well as start-up costs. In the case presented here, in comparison to the "most correct" model (i.e., the UC model), the operational costs of the TIMES model are shown to be underestimated by $3-53 \%$, where higher deviations correspond to higher shares of IRES. Again, it can be observed that both the temporal representation, and the low level of techno-economic operational detail contribute to this underestimation of operational costs.

\begin{tabular}{|c|c|c|c|c|c|}
\hline & 2014 & 2020 & 2030 & 2040 & 2050 \\
\hline Operational cost TIMES [EUR/MWh] & 29.9 & 25.2 & 13.1 & 10.9 & 9.4 \\
\hline Operational cost MO dispatch [EUR/MWh] & 30.0 & 25.3 & 14.5 & 15.5 & 17.5 \\
\hline Operational cost UC [EUR/MWh] & 30.7 & 26.0 & 16.8 & 18.6 & 20.1 \\
\hline$\overline{I m p a c t}$ temporal representation $[\overline{\mathrm{E}} \overline{\mathrm{U}} \overline{\mathrm{R}} / \overline{\mathrm{M}}$ & 0.1 & 0.1 & 1.4 & 4.6 & $8.1^{-}$ \\
\hline Impact techno-economic detail [EUR/MWh] & 0.7 & 0.7 & 2.3 & 3.1 & 2.6 \\
\hline
\end{tabular}

Table 6: Operational costs in the different models. All costs are expressed relative to the total consumed electric energy.

When these model simplifications are applied in models used for analyzing transition pathways towards 
more sustainable energy systems with high shares of IRES, all the above-mentioned errors occur simultaneously. That is, the operational costs are underestimated, the uptake of IRES will be overestimated and the level of baseload generation will be overestimated. As a result, the efforts needed to effectively obtain a desired reduction of GHG emissions (or RES penetration) are likely to be underestimated considerably. Moreover, models using these model simplifications tend to overly value intermittent and inflexible generation, while not providing sufficient incentives for different flexibility options (e.g., flexible generation, storage technologies, grid expansions, demand side management). This is particularly important as this type of models commonly serves as a base for underpinning policy regarding R\&D of innovative energy technologies. It must therefore be concluded that the model simplifications typically used in long-term planning models can no longer be justified when analyzing systems with high shares of IRES.

\subsection{Level of temporal versus techno-economic operational detail}

To improve these planning models, one could either adapt the temporal representation or increase the level of techno-economic operational detail. This section analyzes the impact of the individual model simplifications, thereby aiming to provide guidelines as to which aspect should be addressed with the highest priority.

From Tables 4 and 6, it can be seen that for a low penetration of IRES, the impact of the level of techno-economic detail is higher than the impact of the temporal representation, both in terms of impact on the generation mix and impact on the operational cost. However, for penetration levels of IRES up to $12.5 \%$ in terms of yearly electric energy generated, the impact of both model simplifications is limited. When the IRES penetration reaches a level of $25 \%$, the impact of both model simplifications has risen significantly, and is similar in terms of impact on the generation mix. Regarding the operational cost, the level of techno-economic detail has the highest impact on the results. However, for even higher shares of IRES, the impact of the temporal representation increases strongly, while the impact of the low level of techno-economic detail starts to stagnate and even decreases. As a result, for high penetrations of IRES, the temporal representation becomes the dominant factor.

The impact of the temporal representation can be explained by analyzing how it accounts for the variability of IRES generation. Figure 4 displays how temporal representation 'Integral TS low' approximates the wind generation duration curve and the residual load duration curve (RLDC). Different authors have highlighted the significance of the RLDC for the investment planning problem 32, 33, 34. The residual load for each time slice is found by subtracting the potential undispatchable electricity generation in that time slice from the load corresponding to that time slice 6 . Sorting this residual load from high to low gives the RLDC. This RLDC contains information about key aspects related to the integration of IRES, such as the low capacity credit of IRES, the reduction of operating hours of thermal power plants, and the amount of excess energy (possibly leading to curtailment) 32. To capture these characteristics of IRES, it is therefore important that the temporal representation approximates the RLDC with high accuracy.

Figure 4 clearly illustrates that, while temporal representation 'Integral TS low' approximates the load duration curve with reasonable accuracy, this is not the case for the wind generation duration curve (and hence not for the RLDC). After all, the idea behind this temporal representation is based on capturing the significant seasonal and diurnal differences between each time slice, on the one hand, and the similarities of data values that belong to a specific time slice (e.g., electricity demand in peak periods during the winter), on the other hand [21. As the load profile has strong regularities on the seasonal, daily and diurnal level, time-slice divisions such as 'Integral TS low' obtain a good representation of the load profile. In contrast, due to the lack of regularities in wind-power fluctuations, averaging the wind-turbine electricity generation data belonging to a specific time slice (e.g., all wind data corresponding to periods of peak demand during the entire winter) causes periods of very high or very low wind generation to be overlooked. This is reflected in the relatively flat approximated wind generation duration curve.

${ }^{6}$ The only undispatchable electricity generation considered is the electricity generation by wind turbines and solar PV panels. Moreover, the term "potential electric energy generation", as opposed to the actual electric energy generation, is used to account for the fact that the actual electric energy generation can be lower than the potential electric energy generation in a specific time slice in case of curtailment. 

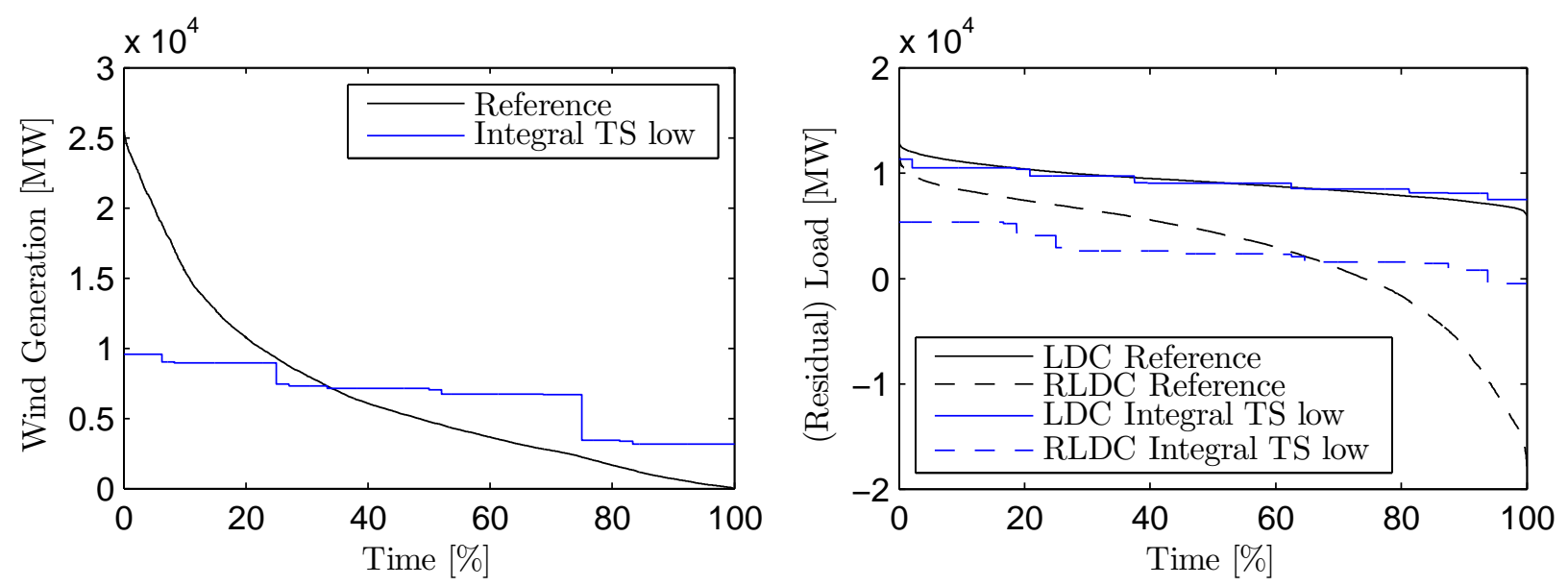

Figure 4: Impact of the temporal representation on the approximation of the wind generation duration curve (left figure) and the (residual) load duration curve (right figure) for model year 2050. The reference corresponds to the sorted hourly data for an entire year.

As can be observed in Figure 4 this will yield an approximation of the RLDC which is too flat. On the one hand, this causes a considerable underestimation of the peak residual load as these peaks occur when IRES power generation is very low and the load is high, i.e., the capacity credit of IRES is overestimated. In this regard, the role of the constraint demanding sufficient firm capacity is crucial to obtain generation portfolios which can achieve a reasonable security of supply. On the other hand, the residual load in periods of high IRES generation is overestimated. This results in an overestimation of the number of full load hours that can be obtained by baseload technologies. Moreover, periods of excess electric energy generation might be overlooked, causing an overestimation of the potential uptake of IRES electricity generation. Both effects can be observed in Figure 3. In terms of costs, this results in an underestimation of operational costs, as overestimating the share of baseload generation and IRES generation leads to an underestimation of electricity generation by more expensive mid-merit and peak-load technologies (see Table 6). As can be observed from Tables 4 and 6 . the impact of a temporal representation that does not properly account for the variability of IRES generation grows strongly with the share of IRES.

The impact of the level of techno-economic operational detail is more complex for multiple reasons. First, what is referred to as the level of techno-economic operational detail bundles a variety of different technical constraints (e.g., ramping rate restrictions, minimum up and down times) as well as economic operational characteristics (e.g., start-up costs, part-load efficiency losses), all having a different impact. Second, in contrast with the impact of the temporal representation, the penetration level of IRES is no longer the only driver for differences in the dispatch. That is, also the flexibility of the thermal generation fleet plays a role. Whereas the penetration of IRES determines the volatility of the residual load, the possibilities to satisfy this fluctuating residual load are determined by the flexibility of the generation fleet. Moreover, in the long 320 term, the penetration of IRES and the flexibility of the thermal generation fleet are interdependent. With an increasing share of IRES, the number of full load hours of baseload technologies is reduced. Therefore, one could expect that the fraction of baseload capacity, which is generally less flexible, is reduced as the penetration of IRES increases over time. As a result, the generation fleet will likely become more flexible as the penetration of IRES increases. This can also be observed in the results of the planning model from 2030 on, where the installed capacity of nuclear plants is reduced (see Table 4). Before 2030, the nuclear capacity is increased despite the fact that there is an increasing penetration of IRES. This is due to the fact that the model starts from the existing Belgian capacity mix which, according to the data used in this work, contains a suboptimally low level of nuclear plants. Due to the lead times of building new plants, and the gradual retirement of existing capacity, the capacity of nuclear plants cannot instantly be increased. 
If the penetration of IRES is low, it can be observed from Table 4 that the level of techno-economic operational detail has little impact on the generation mix. This is due to the fact that the variability of the residual load is still limited, and the technical constraints do not become binding, i.e., there is excess flexibility. However, in terms of operational costs, there will always be plants which need to cycle (i.e., change the power output by ramping up/down or by switching on/off), thereby incurring start-up costs, ramping costs and additional fuel and emission costs due to efficiency losses in part-load operation. Up to 2020 , these costs are responsible for over $50 \%$ of the underestimation of the operational costs due to the low level of techno-economic detail 7

As the penetration of IRES, and therefore, the variability of the residual load, increases, there will be an increased need for cycling. Moreover, due to the large fraction of electric power generated by IRES in some moments, there will be an increased need for cycling of baseload and mid-merit power plants. On the one hand, these baseload plants are generally less flexible, and the technical constraints for these plants can become binding. In these cases, the dispatch proposed by the TIMES model and the MO dispatch model might be technically infeasible, and the UC model will be forced to schedule additional flexible generation and/or to curtail IRES generation. The result of neglecting these technical constraints is an overestimation of the generation by baseload technologies and an overestimation of the uptake of IRES generation. On the other hand, the dispatch of the UC model might deviate from the dispatch in the MO model to avoid start-up costs, i.e., the dispatch proposed by the MO model might not be optimal when start-up costs are accounted for. Regarding the uptake of IRES generation, this might lead to additional curtailment, as it can be more economic to curtail some IRES generation, than to shut down a plant which would have to be brought on line again some hours later. Regarding baseload generation, the impact of accounting for start-up costs is twofold. Baseload plants might be kept on line to prevent start-up costs some hours later, thereby leading to curtailment of IRES. Alternatively, baseload plants might be kept off line to avoid the high start-up costs. For the year 2030, the impact of the level of techno-economic operational detail has increased significantly with respect to the first two periods. This is due to the fact that both the IRES penetration and the share of baseload capacity have increased with respect to 2014 . While for the first two periods, hardly any impact on the generation shares could be observed, this is no longer the case in 2030 . In terms of operational costs, a strong increase in the underestimation of operational costs can be observed. Whereas, the start-up costs, ramping costs and costs related to part-load efficiency losses were responsible for over $50 \%$ of the underestimation of operational costs in the first two periods, this share has dropped to less than $20 \%$ in 2030 , indicating that it is mainly the switch to an increased generation by more flexible, but more expensive CCGT's, which has caused the increased underestimation of operational costs.

Further increasing the share of IRES will further increase the need for cycling. However, it can be observed that, from 2040 onwards, the capacity of less-flexible nuclear capacity is reduced. As a result, the impact of the level of techno-economic operational detail starts to stagnate, and is even slightly reduced by 2050 (see Tables 4 and 6). In this regard, it must be noted that the capacity mix used as input in the UC model corresponds to the solution of a planning model with temporal representation 'Integral TS low', which, as discussed above, causes to overestimate the potential of baseload technologies. Therefore, the impact of the low level of techno-economic operational detail would likely be even lower when the temporal representation would be improved. Moreover, CHP plants and small generators were not considered in the 370 analysis. Nevertheless, these types of generation can provide a significant source of generation flexibility and could therefore reduce the impact of the low level of technical detail even further.

It can be concluded that for a low penetration of IRES, both model simplifications have a limited impact on the results. As the penetration of IRES increases, also the impact of the temporal representation and the low level of techno-economic operational detail start to increase. However, whereas the impact of the low level of techno-economic operational detail starts to stagnate for even higher penetrations of IRES (as a high penetration of IRES gives rise to a more flexible generation fleet), the impact of the temporal representation

${ }^{7}$ This only includes the direct costs related to start-ups, ramping and part-load efficiency losses. In some situations, the knowledge of start-up and ramping costs will incur indirect costs, e.g., more expansive generation can be brought/kept on line to avoid the high start-up costs for bringing on line a large baseload/mid-merit unit when little additional capacity is required. Such indirect costs are not included here. 
keeps increasing. For IRES penetration levels of 35-50\%, in terms of yearly electric energy generated, the impact of the temporal representation is shown to be significantly higher than the impact of the level of techno-economic operational detail, which seems to be in line with the results presented in [16. For this reason, improving the temporal representation in planning models is suggested to be prioritized.

\subsection{Improving the temporal representation}

This section aims to identify opportunities for improving the temporal representation in LT planning models. As discussed in Section 1, much attention has recently gone to the temporal resolution used in planning models. However, it is not merely the resolution (i.e., the number of diurnal time slices) which is of importance. Less discussed in the scientific literature, though equally important, is how the set of time slices is chosen (i.e., the general structure of the time-slice tree) and the approach used to assign values to the load and IRES availability in each time slice.

Regarding the approach used to assign values to each time slice, the integral method is most commonly applied. As discussed in Section 4.2, using this approach for a limited number of time slices can lead to averaging of IRES generation, thereby strongly underestimating the variability of IRES generation. An alternative approach to assign a value to each time slice is to select a set of historical days (or weeks) to represent an entire year. Each selected day/week represents a part of the year (e.g., a season or a month). These representative days/weeks can in turn be divided into a number of diurnal time slices. As such, the value assigned to each time slice is not the result of taking an average over multiple days, as is the case for the integral method. Depending on the number of days/weeks selected and the diurnal resolution, the total number of time slices used in this approach is typically somewhat higher (16-288).

To illustrate the difference between the integral method and the approach based on selecting representative days, consider a model with 4 seasonal time slices and 3 diurnal time slices (day, night and peak). Figure 5 visualizes the process of assigning a value to the time slice representing periods of peak demand during

400 the winter, for both approaches. The graphs on the left hand side show profiles of the load, the residual load and onshore wind generation during a winter week for an exemplary system with a high penetration of wind power. Every day, samples are taken during the peak hours (lasting 2 hours in each day). The graphs on the right hand side display all the samples taken during the entire winter season (91 days). The blue lines indicate the values assigned to the winter peak time slice in the integral method, i.e. the average of all 405 samples. Following the method using representative days, the value assigned to the winter peak time slice corresponds to the average value of the samples corresponding to the selected day. Hence, the value can lie anywhere in the shaded intervals, depending on the selected day. The red line indicates the value assigned to this time slice if the sample displayed in red would be selected.

Visualizing the processes of assigning a value to a specific time slice highlights some of the main advantages and drawbacks of each method. The main drawback of the integral method is that the range of IRES generation levels occurring in reality is not retained as the average value of all samples belonging to this time slice is taken. Therefore, this method does not capture periods with very high or very low IRES generation, as was shown in Figure 4. In contrast, when using representative days, the IRES generation can be anywhere within the range actually observed. However, the main issue with this approach is the difficulty of selecting a set of days which is representative for an entire year, or multiple years. Figure 5 illustrates the sensitivity of this method to the selected representative day. Nonetheless, de Sisternes and Webster remark that there is no consistent criterion to select days/weeks or to assess the validity of the assumption [35. However, recently, different authors have focused on specific algorithms and models to select a good set of representative days 36,37 .

To improve the temporal representation, different alternative temporal representations can be thought of, either by adapting the structure of the time-slice tree, the way values are assigned to each time slice, or both. In the further analysis, 3 different approaches are considered. The details of each considered temporal representation are presented in Table 7. Regarding the temporal structure, a different lay-out of the time-slice tree can be considered to improve capturing IRES variability in the integral approach. The approach which has received the most attention in the scientific literature is to increase the temporal resolution (i.e., the number of diurnal time slices in the time-slice tree) ('Integral TS high'). An alternative approach is to devote a separate level in the time-slice tree to explicitly distinguish periods of high and low 

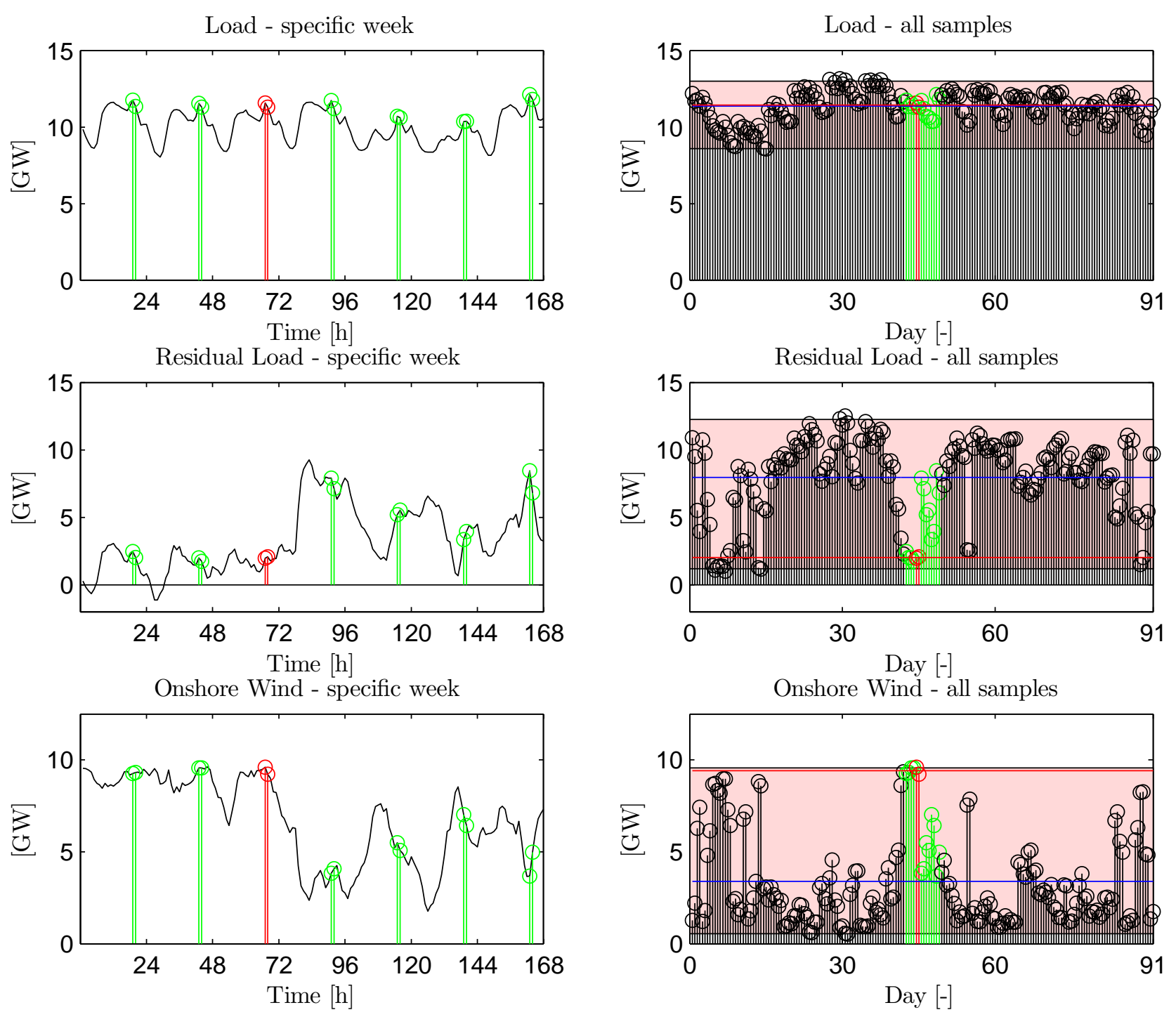

Figure 5: Process of assigning values to time slices using the integral method and the approach based on using representative days.

resource availability ('Integral TS alt'). This approach still takes the average value of multiple samples taken from IRES profiles in different historical days, but now the average is only taken over a subset of samples with similar resource availabilities (e.g., the $20 \%$ of samples corresponding to the highest wind generation). Finally, a set of representative days could be used to assign values to the different time slices ('Repr. days TS high').

To evaluate these temporal representations, the RLDC is constructed for an increasing penetration of wind turbines, and compared to the RLDC based on an entire year of hourly data. The root-mean-square error (RMSE) relative to the peak load is used as a metric for evaluating the quality of the approximation.

Figure 6 displays for each considered temporal representation the accuracy of the approximation of the RLDC as a function of the wind power penetration. This figure shows that, relative to temporal representation 'Integral TS low', increasing the resolution in the integral approach ('Integral TS high') 


\begin{tabular}{|c|c|c|c|c|c|}
\hline \multirow{2}{*}{$\begin{array}{l}\text { Temporal } \\
\text { representation }\end{array}$} & \multicolumn{5}{|c|}{ Number of time slices } \\
\hline & Seasonal & Daily & Diurnal & IRES & Total \\
\hline Integral TS low & 4 & - & 3 (day, night, peak) & - & 12 \\
\hline Integral TS high & 4 & 3 (Weekday, Sat, Sun) & 24 & - & 288 \\
\hline Integral TS alt & 4 & - & 3 (day, night, peak) & 3 (high, medium, low) & 36 \\
\hline Repr. days TS high & 4 & 3 (Weekday, Sat, Sun) & 24 & - & 288 \\
\hline $\bar{R}{ }^{-}-{ }^{-}-{ }^{-}-{ }^{-}--$ & $\overline{5} 2^{-}$ & $-\frac{5}{7}$ & $\overline{24}$ & $\overline{-}$ & $\overline{8} 7 \overline{3} \overline{6}$ \\
\hline
\end{tabular}

Table 7: Overview of the different temporal representations considered. This table indicates the number of levels and branches in the time-slice tree. The terms "Integral" and "Repr. days" refer to the methodology used for assigning values to each time slice.

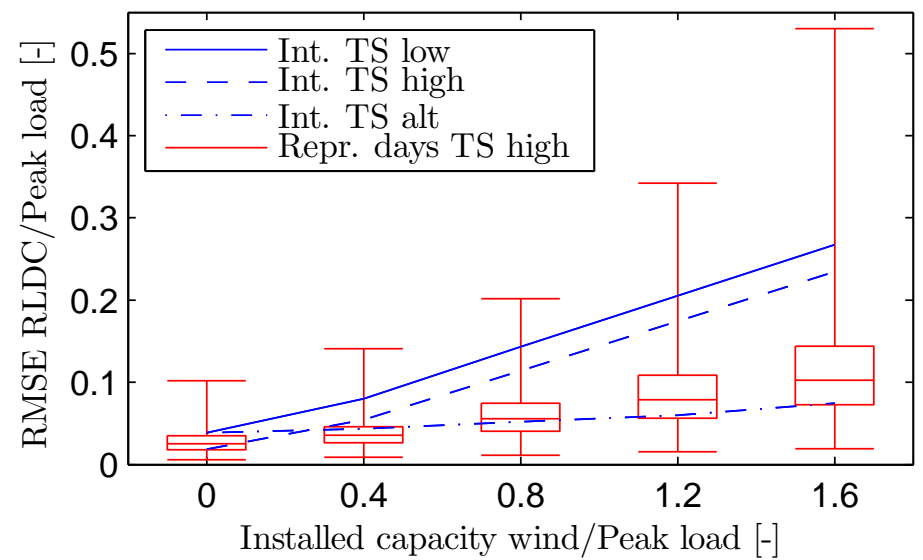

Figure 6: Approximation error of the residual load duration curve (RLDC) for different temporal representations and a varying wind power penetration.

yields limited benefits. These benefits are predominantly due to a better approximation of variations in the load. As can be seen, the gain in accuracy compared to the case 'Integral TS low' remains almost constant as the wind power penetration is increased. This indicates that increasing the resolution in the integral approach brings about no additional benefits related to grasping IRES variability. This is due to the fact that the value for wind generation in each time slice is found by taking the average wind generation of all data samples corresponding to this time slice. Due to the higher resolution, the average is taken over a lower number of samples than is the case for temporal representation 'Integral TS low'. However, already by taking the average value over a small set of samples, wind generation is smoothed out. Opposite effects can be observed for temporal representation 'Integral TS alt'. Adding a time slice level to explicitly account for variations in IRES availability brings about no advantage for approximating load variations, but, as the share of IRES is increased, drastically improves the approximation of the RLDC. This illustrates that by a different choice of the structure of the time-slice tree, results can be significantly improved, with only a limited increase in the number of time slices. Finally, using a number of representative days ('Repr. days TS high') can yield an even higher accuracy of the RLDC approximation, provided a good selection of representative days is made ${ }^{8}$ This is highlighted by the box plots which correspond to 10000 random selections of 1 week day, 1 Saturday and 1 Sunday per season (each day is appropriately weighted). The median, the $25^{\text {th }}$ and $75^{\text {th }}$ percentile (rectangle), as well as the highest and lowest errors obtained (whiskers) are shown. The large spread in the accuracy obtained using this approach highlights the importance of a good selection of representative days.

\footnotetext{
${ }^{8}$ It must be noted that the RLDC approximation in the approach using a separate level in the time-slice tree for IRES can be improved by increasing the number of time slices.
} 
As discussed in Section 4.2 the RLDC provides valuable information with respect to capturing the characteristics of intermittent generation. However, the approximation of the RLDC does not tell the entire story. First, the RLDC does not provide information about the dynamic fluctuations of the residual load. Second, the RLDC does not contain information about the individual elements used to construct this curve, i.e., a temporal representation that results in a good approximation of the RLDC does not necessarily provide a good approximation of the load duration curve, the wind generation duration curve or the solar PV generation duration curve. Nevertheless, both these aspects are of importance to assess the value of different technologies in the energy system. Capturing the short-term (ST) dynamic fluctuations will determine the arbitrage opportunities, and hence the value of flexibility options, such as storage technologies, flexible power plants, active demand response and increased interconnection capacity. Moreover, an erroneous representation of the wind generation duration curve or solar PV generation duration curve can lead to a technology bias for two reasons. On the one hand, this can result from an overestimation/underestimation of the yearly generation of wind turbines or solar PV panels. On the other hand, this can also be due to the shape of the wind generation duration curve or solar PV generation duration curve. It is therefore important to consider to what extent the different temporal representations capture these aspects. Finally, also the number of time slices is of importance as this directly impacts the computational cost. An overview of the performance of the different temporal representations on these different aspects is presented in Table 8

\begin{tabular}{lcccc}
\hline \hline & Int. TS low & Int. TS high & Int. TS alt & Repr. days TS high \\
\hline Approximation RLDC & - & - & + & ++ \\
Capturing ST dynamics & - & - & - & + \\
$-{ }_{-}-\overline{-}_{-}-{ }_{-}$ & + & - & + & + \\
Computational requirements & ++ & - & + & - \\
\hline \hline
\end{tabular}

Table 8: Overview of the strengths and weaknesses of the different time-slice approaches.

By definition, the temporal representations using the integral method provide a correct value for the yearly generation of wind turbines and solar PV panels. Moreover, by explicitly defining periods of high and low resource availability, the shape of the wind turbine generation duration curve and solar PV generation duration curve can be approximated with a reasonable accuracy. However, this is not necessarily the case for a temporal representation based on selecting representative days. Care is needed in selecting a set of representative days that not only provides a good approximation of the RLDC, but also correctly represents the different resource availabilities. Indeed, even a set of representative days that obtains a low error in approximating the RLDC can lead to a significant technology bias, and is therefore not applicable. This again stresses the fact that the quality of the approach based on using representative days strongly depends on the choice of the set of representative days. Selecting a good set of representative days requires implementation of a separate algorithm or optimization model. As a result, this approach can require more effort than the 'integral' approaches.

Regarding the ST dynamic fluctuations, it is shown that temporal representations such as 'Integral TS low' and 'Integral TS high' underestimate the range of IRES generation levels (see Figure 4). Therefore, these temporal representations will also underestimate short-term dynamic variations in the residual load, and nce the value of the different flexibility options. In contrast, a temporal representation such as 'Integral TS alt' does capture periods of high and low wind generation. However, such a temporal representation does not preserve chronology, and does therefore not provide information about the frequency of these variations or the time scales at which these variations take place. As a result, this temporal representation might not be suited to assess the role of time-constrained flexibility options, such as storage technologies and active demand response. Finally, by using representative days, periods of high and low wind generation can occur and, within each representative day, chronology is retained. Consequently, a temporal representation such as 'Repr. days TS high' is likely to be most suited to capture ST dynamic fluctuations and the value of different flexibility options. It must be noted that the true value of these flexibility options might only become apparent when a high level of techno-economic detail is used. Some of the techno-economic operational constraints, such as ramping rate restrictions, minimum up and down times and start-up costs, can only 
be modeled in detail by linking sequential time slices. Therefore, chronological data (at a sufficiently high resolution) is a prerequisite for modeling these constraints. As a result, a model with temporal representation such as 'Integral TS alt' cannot be extended to incorporate a high level of techno-economic detail, whereas this would be possible for a model with temporal representation 'Repr. days TS high'.

In conclusion, both a temporal representation which uses a separate level in the time-slice tree for IRES availability, and a temporal representation based on using a set of representative days can strongly improve the way LT energy system planning models represent IRES. The main drawback of using a separate time-slice level for IRES generation is that chronology is not preserved. Therefore, this approach might not be suited to assess the impact of short-term dynamic variations of IRES generation, and the potential/requirement of different flexibility options to deal with these variations. In contrast, using a temporal representation based on using a set of representative days retains chronology (intra-daily), and is therefore better suited to assess the potential of different flexibility options. Moreover, this approach is able to provide a better approximation of the RLDC, albeit using a higher number of time slices (and therefore a higher computational cost). The main drawback of this approach is the difficulty of selecting a good set of representative days.

\section{Summary and conclusions}

Long-term energy-system planning models are frequently used in studies analyzing the transition towards a sustainable energy system. In these studies, intermittent renewable energy sources (IRES) are expected to be key contributors to this transition. However, their highly variable and stochastic nature poses challenges to long-term energy-system planning models, as these models typically use a low level of temporal and

520 techno-economic operational detail. In this regard, closing the gap between short-term operational models and long-term planning models has become an active field of research.

This paper provides a comprehensive analysis of how the operational dimension of the electric power system can impact the results of system planning models. A detailed modeling analysis is set-up to quantify the impact of both the temporal and techno-economic representation typically used in these models for an 525 increasing penetration of IRES. This allows making the trade-off between improving planning models by extending the operational time dimension, and/or aiming for a better technical representation. Both the temporal and techno-economic operational representation are shown to have the same qualitative impact on the results, i.e., a low level of detail results in an overestimation of the potential uptake of IRES, an overestimation of the generation by baseload technologies and an underestimation of operational costs. While for a low penetration of IRES, the impact of both the temporal representation, and the low level of technoeconomic operational detail is limited, the impact of the temporal representation becomes dominant for a high share of IRES electric energy generation (35-50\%). Prioritizing addressing the temporal representation is therefore recommended.

Different approaches to improve the temporal representation in planning models are proposed and evaluated by analyzing how well the residual load duration curve is approximated for a varying penetration of IRES. In this regard, not only the structure of the time-slice tree, but also the method of assigning values to each time slice is shown to be of importance. Using a time-slice level to explicitly account for different levels of IRES availability improves the approximation of the RLDC significantly, without drastically increasing the number of time slices. However, this approach does not preserve chronology, making it less suited to assess the potential of different (time-constrained) flexibility options. On the other hand, using a temporal representation based on a set of representative days can also result in a drastic increase in accuracy, while retaining chronology intra-daily. However, this approach requires a larger number of time slices and care is needed in selecting an appropriate set of representative days.

Further research involves evaluating the proposed approaches by using the presented temporal representations in a LT investment planning model. Regarding the use of a temporal representation based on using representative days, more research is needed to analyze the potential of using a low number of representative days. Moreover, a consistent criterion for selecting representative days is needed. Finally, the impact of including sources of dispatchable distributed generation, such as combined heat and power plants and small generators, on the impact of the low level of techno-economic operational detail is left for future research. 
To conclude, some guidelines that can be considered when performing a scenario analysis using a bottomup long-term energy system planning model are presented:

- When setting up or revising the temporal representation of the model, evaluating how this temporal representation approximates the RLDC should be considered. Nevertheless, this approach has some limitations of which the modeler must be aware. First, due to the loss of chronology, the RLDC might be approximated with high accuracy by the temporal representation, while the dynamics of the variations of the residual load might not be grasped. Second, information regarding the individual resource availability profiles (e.g., wind, sun) is not provided by the RLDC. To prevent a technology bias, additionally evaluating how the temporal representation approximates the resource availability duration curves should be considered.

- In addition to the temporal structure of the time-slice tree, the process used to assign values to the time slices should be documented. While this information is often lacking, it is proven to be essential for assessing and interpreting the results obtained by these models.

- Improving the temporal representation should be prioritized to increasing the level of techno-economic operational detail.

- The temporal representation can be improved by using a well-chosen set of representative historical days.

- When computational resources are scarce, an alternative can be to deviate from the typical structure of the time-slice tree (i.e., based on time slice levels to account for seasonal, daily and diurnal variations) by using a time slice level that explicitly accounts for IRES availability.

\section{Acknowledgements}

The research of Kris Poncelet is supported by a PhD grant provided by VITO. Erik Delarue is a postdoctoral research fellow of the Research Foundation - Flanders (FWO).

\section{References}

[1] D. Connolly, H. Lund, B. Mathiesen, M. Leahy, A review of computer tools for analysing the integration of renewable 575 energy into various energy systems Applied Energy 87 (4) (2010) 1059 - 1082. doi:http://dx.doi.org/10.1016/j. apenergy.2009.09.026.

URL http://www.sciencedirect.com/science/article/pii/S0306261909004188

[2] D. Devogelaer, J. Duerinck, D. Gusbin, Y. Marenne, W. Nijs, M. Orsini, M. Pairon, Towards 100\% renewable energy in Belgium by 2050, Tech. rep., VITO, ICEDD and the Belgian Federal Planning Bureau (2013).

[3] K. Karlsson, P. Meibom, Optimal investment paths for future renewable based energy systems - using the optimisation

model balmorel, International Journal of Hydrogen Energy 33 (7) (2008) 1777 - 1787. doi:http://dx.doi.org/10.1016/ j.ijhydene.2008.01.031

URL http://www.sciencedirect.com/science/article/pii/S0360319908000888

[ [4] A. Chiodi, M. Gargiulo, F. Rogan, J. Deane, D. Lavigne, U. K. Rout, B. P. . Gallachóir, Modelling the impacts of challenging 2050 european climate mitigation targets on ireland's energy system Energy Policy 53 (0) (2013) 169 - 189. doi:http://dx.doi.org/10.1016/j.enpol.2012.10.045

URL http://www.sciencedirect.com/science/article/pii/S0301421512009263

[5] N. Strachan, N. Balta-Ozkan, D. Joffe, K. McGeevor, N. Hughes, Soft-linking energy systems and \{GIS\} models to investigate spatial hydrogen infrastructure development in a low-carbon $\{\mathrm{UK}\}$ energy system International Journal of Hydrogen Energy 34 (2) (2009) 642 - 657. doi:http://dx.doi.org/10.1016/j.ijhydene.2008.10.083. URL http://www.sciencedirect.com/science/article/pii/S036031990801402X

[6] D. Martinsen, J. Linssen, P. Markewitz, S. Vögele, CCS: A future CO2 mitigation option for germany? - a bottom-up approach Energy Policy 35 (4) (2007) 2110 - 2120. doi:http://dx.doi.org/10.1016/j.enpol.2006.06.017 URL http://www.sciencedirect.com/science/article/pii/S030142150600276X

[7] D. Most, M. Genoese, A. Esser, O. Rentz, European electricity and emission market modeling ; the design of emission allocation plans and its effects on investment planning, in: Electricity Market, 2008. EEM 2008. 5th International Conference on European, 2008, pp. 1-6. doi:10.1109/EEM.2008.4579087 
[8] G. Luderer, V. Krey, K. Calvin, J. Merrick, S. Mima, R. Pietzcker, J. Van Vliet, K. Wada, The role of renewable energy in climate stabilization: results from the EMF27 scenarios, Climatic Change 123 (3-4) (2014) 427-441. doi: $10.1007 / \mathrm{s} 10584-013-0924-\mathrm{z}$ URL http://dx.doi.org/10.1007/s10584-013-0924-z

[9] D. Lew, G. Brinkman, E. Ibanez, A. Florita, M. Heaney, B.-M. Hodge, M. Hummon, G. Stark, J. King, S. Lefton, N. Kumar, D. Agan, G. Jordan, S. Venkataraman, The western wind and solar integration study phase 2, Tech. rep., National Renewable Energy Laboratory (NREL) (2013).

[10] K. Van den Bergh, E. Delarue, W. D'haeseleer, The impact of renewable injections on cycling of conventional power plants, in: European Energy Market (EEM), 2013 10th International Conference, 2013, pp. 1-8. doi:10.1109/EEM.2013.6607322

[11] K. Van den Bergh, E. Delarue, Facilitating variable generation of renewables by conventional power plant cycling, in: 9th Conference on Energy Economics and Technology (ENERDAY), 2014

[12] M. Milligan, P. Donohoo, D. Lew, E. Ela, B. Kirby, H. Holttinen, E. Lannoye, D. Flynn, M. O'Malley, N. Miller, P. B. Eriksen, A. Gottig, B. Rawn, M. Gibescu, E. G. Lázaro, A. Robitaille, I. Kamwa, Operating reserves and wind power integration: An international comparison, Tech. rep., NREL (2010).

[13] K. Bruninx, E. Delarue, W. D'haeseleer, The cost of wind power forecast errors in the belgian power system, in: IAEE, 37th international conference, 2014.

[14] B. Palmintier, M. Webster, Impact of unit commitment constraints on generation expansion planning with renewables, in: Power and Energy Society General Meeting, 2011 IEEE, 2011, pp. 1-7. doi:10.1109/PES.2011.6038963

[15] S. Pfenninger, A. Hawkes, J. Keirstead, Energy systems modeling for twenty-first century energy challenges, Renewable and Sustainable Energy Reviews 33 (0) (2014) 74-86. doi:http://dx.doi.org/10.1016/j.rser.2014.02.003 URL http://www.sciencedirect.com/science/article/pii/S1364032114000872

[16] J. Deane, A. Chiodi, M. Gargiulo, B. P. . Gallachóir, Soft-linking of a power systems model to an energy systems model 620 Energy 42 (1) (2012) 303 - 312, 8th World Energy System Conference, \{WESC\} 2010. doi:http://dx.doi.org/10.1016/ j.energy.2012.03.052

URL http://www.sciencedirect.com/science/article/pii/S0360544212002551

[17] J. Rosen, I. Tietze-Stöckinger, O. Rentz, Model-based analysis of effects from large-scale wind power production Energy 32 (4) (2007) 575 - 583, eCOS 05. 18th International Conference on Efficiency, Cost, Optimization, Simulation, and Environmental Impact of Energy Systems ECOS 05. doi:http://dx.doi.org/10.1016/j.energy.2006.06.022 URL http://www.sciencedirect.com/science/article/pii/S0360544206001605

[18] A. Pina, C. A. Silva, P. Ferrão, High-resolution modeling framework for planning electricity systems with high penetration of renewables, Applied Energy 112 (0) (2013) 215 - 223. doi:http://dx.doi.org/10.1016/j.apenergy.2013.05.074 URL http://www.sciencedirect.com/science/article/pii/S030626191300487X

630 [19] S. Ludig, M. Haller, E. Schmid, N. Bauer, Fluctuating renewables in a long-term climate change mitigation strategy Energy 36 (11) (2011) 6674 - 6685. doi:http://dx.doi.org/10.1016/j.energy.2011.08.021 URL http://www.sciencedirect.com/science/article/pii/S0360544211005512

[20] G. Haydt, V. Leal, A. Pina, C. A. Silva, The relevance of the energy resource dynamics in the mid/long-term energy planning models Renewable Energy 36 (11) (2011) 3068-3074. doi:http://dx.doi.org/10.1016/j.renene.2011.03.028 URL http://www.sciencedirect.com/science/article/pii/S096014811100142X

[21] A. Pina, C. Silva, P. Ferrão, Modeling hourly electricity dynamics for policy making in long-term scenarios, Energy Policy 39 (9) (2011) 4692 - 4702. doi:http://dx.doi.org/10.1016/j.enpol.2011.06.062 URL http://www.sciencedirect.com/science/article/pii/S0301421511005180

[22] M. Welsch, P. Deane, M. Howells, B. . Gallachóir, F. Rogan, M. Bazilian, H.-H. Rogner, Incorporating flexibility requirements into long-term energy system models - a case study on high levels of renewable electricity penetration in ireland, Applied Energy 135 (0) (2014) 600 - 615. doi:http://dx.doi.org/10.1016/j.apenergy.2014.08.072 URL http://www.sciencedirect.com/science/article/pii/S0306261914008836

[23] R. Kannan, H. Turton, A long-term electricity dispatch model with the TIMES framework Environmental Modeling \& Assessment 18 (3) (2013) 325-343. doi:10.1007/s10666-012-9346-y URL http://dx.doi.org/10.1007/s10666-012-9346-y

[24] C. Nweke, F. Leanez, G. Drayton, M. Kolhe, Benefits of chronological optimization in capacity planning for electricity markets, in: Power System Technology (POWERCON), 2012 IEEE International Conference on, 2012, pp. 1-6. doi: 10.1109/PowerCon.2012.6401421

[25] R. Loulou, U. Remne, A. Kanudia, A. Lehtila, G. Goldstein, Documentation for the TIMES model: Part I, ETSAP (April 2005).

[26] K. Van den Bergh, K. Bruninx, E. Delarue, W. D'haeseleer, Lusym, a unit commitment model formulated as a mixedinteger linear program (July 2015).

[27] S. Simoes, W. Nijs, P. Ruiz Castello, A. Sgobbi, P. Radu, Daniela abd Bolat, C. Thiel, E. Peteves, The JRC-EU-TIMES model - assessing the long-term role of the SET plan energy technologies, Tech. rep., JRC's Institute for Energy and Transport (2013).

[28] Projected costs of generating electricity - 2010 edition, Tech. rep., IEA and NEA (2010).

[29] A. Schröder, F. Kunz, J. Meiss, R. Mendelevitch, C. von Hirschhausen, Current and prospective costs of electricity generation until 2050, Tech. rep., DIW Berlin (2013).

[30] Elia grid data (July 2014). URL http://www.elia.be/en/grid-data

[31] World energy outlook 2013, Tech. rep., IEA (2013).

[32] F. Ueckerdt, R. Brecha, G. Luderer, P. Sullivan, E. Schmid, N. Bauer, D. Böttger, Variable renewable energy in modeling 
climate change mitigation scenarios, in: International Energy Workshop, 30th edition, Stanford, 2011.

[33] C. D. Jonghe, E. Delarue, R. Belmans, W. D'haeseleer, Determining optimal electricity technology mix with high level of wind power penetration Applied Energy 88 (6) (2011) 2231 - 2238. doi:http://dx.doi.org/10.1016/j.apenergy.2010. 12.046

URL http://www.sciencedirect.com/science/article/pii/S0306261910005660

[34] S. Stoft, Power System Economics: Designing Markets for Electricity, IEEE Press and WILEY-INTERSCIENCE, 2002.

[35] F. J. de Sisternes, M. D. Webster, Optimal selection of sample weeks for approximating the net load in generation planning problems (March 2013).

P. Nahmmacher, E. Schmid, L. Hirth, B. Knopf, Carpe Diem: A Novel Approach to Select Representative Days for LongTerm Power System Models with High Shares of Renewable Energy Sources, SSRN Journaldoi:10.2139/ssrn.2537072 URL http://dx.doi.org/10.2139/ssrn.2537072

[37] K. Poncelet, H. Höschle, E. Delarue, W. D’haeseleer, Selecting representative days for investment planning models, KU Leuven, Division of applied mechanics and energy conversion, Working paper EN2015-10. 


\section{Nomenclature}

\section{List of symbols}

Sets (indices)

$\mathrm{c}$
$\mathrm{p}$
$\mathrm{s}$
$\mathrm{t}$
$\mathrm{v}$

Index for the commodity

Index the process (technology)

Index for the time slice

Index for the milestone year

Index for the year of investment

Parameters

$A C F R_{p, v, t}$

$A F_{p, v, t, s}$

$C A P_{-} A C T_{p}$

$C O M_{-} F R_{c, s}$

$D E M_{c}$

$F R_{s}$

Fraction of the capacity of a process $(\mathrm{p}, \mathrm{v})$ that is available in year $\mathrm{t}$

Availability factor of a process $(\mathrm{p}, \mathrm{v})$ in time slice $\mathrm{s}$ of year $\mathrm{t}$

Conversion factor from units of capacity to units of activity of process $\mathrm{p}$

Fraction of the yearly demand for commodity c in time slice s

Yearly demand for commodity c

Fraction of the year represented by time slice s

Variables

act $_{p, v, t, s}$

$\operatorname{cap}_{p, v, t}$

flo $o_{c, p, v, t, s, \text { in }^{\prime} /{ }^{\prime} \text { out }}$

Activity (generation) of a process $(\mathrm{p}, \mathrm{v})$ in time slice $\mathrm{s}$ of year $\mathrm{t}$

Installed capacity in year $\mathrm{t}$ of a process $(\mathrm{p}, \mathrm{v})$

Flow of commodity $\mathrm{c}$ in or out of a process $(\mathrm{p}, \mathrm{v})$ in time slice $\mathrm{s}$ of year $\mathrm{t}$

\begin{tabular}{ll}
\multicolumn{2}{l}{ Abbreviations } \\
CCGT & Combined cycle gas turbine \\
CHP & Combined heat and power \\
FOM & Fixed operations and maintenance cost \\
GHG & Greenhouse gas \\
IGCC & Internal gasification combined cycle \\
IRES & Intermittent renewable energy sources \\
LT & Long-term \\
MO & Merit-order \\
OCGT & Open cycle gas turbine \\
PC & Pulverized coal \\
PV & photovoltaic \\
RLDC & Residual load duration curve \\
RMSE & Root-mean-square error \\
SC & Supercritical \\
ST & Short-term \\
SubC & Subcritical \\
TS & Time slice \\
UC & Unit commitment \\
VOM & Variable operations and maintenance cost \\
&
\end{tabular}

